\title{
THE PRE-OPERATIVE RECOGNITION OF THE “MUSCLE-BOUND” RIGHT VENTRICLE IN PULMONARY STENOSIS WITH INTACT VENTRICULAR SEPTUM*
}

\author{
BY

\section{VOGELPOEL, V. SCHRIRE, W. BECK, AND M. NELLEN} \\ From the Cardiac Clinic, Groote Schuur Hospital and C.S.I.R. Cardiovascular-Pulmonary Research Group, Department \\ of Medicine, University of Cape Town, Cape Town, South Africa
}

Received June 27, 1963

In pulmonary stenosis with intact ventricular septum the pre-operative prediction of the severity of the valve stenosis is relatively simple by clinical and hæmodynamic criteria (Vogelpoel and Schrire, 1960). However, the assessment of the degree of secondary muscular hypertrophy in the outflow tract is much more difficult and has received little attention. While likely to be pronounced in all moderate and severe cases, the extent of the hypertrophy differs greatly in subjects with valve stenosis of comparable severity and age. The hypertrophy is sometimes massive and, though apparently innocuous and unobtrusive before operation, presents a major problem by causing severe infundibular stenosis following complete relief of the valve stenosis (Kirklin et al., 1953; Brock, 1955, 1957, 1961 ; Blount et al., 1957; Johnson, 1959). Thus, the pressure in the inflow tract of the right ventricle either fails to fall, or drops inadequately. Such a ventricle has been aptly termed "musclebound " by Brock (1955). When hypertrophy is modest, the right ventricular pressure falls promptly and whatever secondary infundibular stenosis develops is mild and capable of rapid involution.

Hitherto, apart from rapid selective bi-plane angiography or cineangiography (Watson et al., 1960), there have been no reliable signs to enable pre-operative recognition of the degree of subvalvar muscular hypertrophy in a given subject with pulmonary stenosis. However, it is believed that this can be achieved by several new methods presented in this paper.

\section{SubJeCtS AND MethodS}

Although the ideas and observations reported in this paper were formulated from a study of at least 75 patients with pulmonary stenosis with intact ventricular septum, the present analysis is based on 19 patients in whom there was reasonable proof about the degree of secondary muscular hypertrophy in the right ventricular outflow tract. All 19 patients underwent a complete open pulmonary valvotomy.

Group A. There were 11 patients in whom the right ventricle was not considered to be "muscle-bound", because the degree of outflow tract hypertrophy was not excessive on inspection and palpation through the opened pulmonary valve, and the right ventricular systolic pressure fell well below $100 \mathrm{~mm}$. $\mathrm{Hg}$ immediately after valvotomy. A right ventriculotomy and infundibular resection was, therefore, not performed. Two showed moderate secondary infundibular stenosis with relatively low right ventricular systolic pressures (under $75 \mathrm{~mm} . \mathrm{Hg}$ ); they are included because their right ventricles, though hypertrophied, were obviously not "muscle-bound".

* Presented at the South African Cardiac Society Congress held in Durban (1962). A summary of the paper appeared in the South African Medical Journal, 36, 779. 
TABLE I

19 Patients Who Had Complete Open Pulmonary Valvotomy

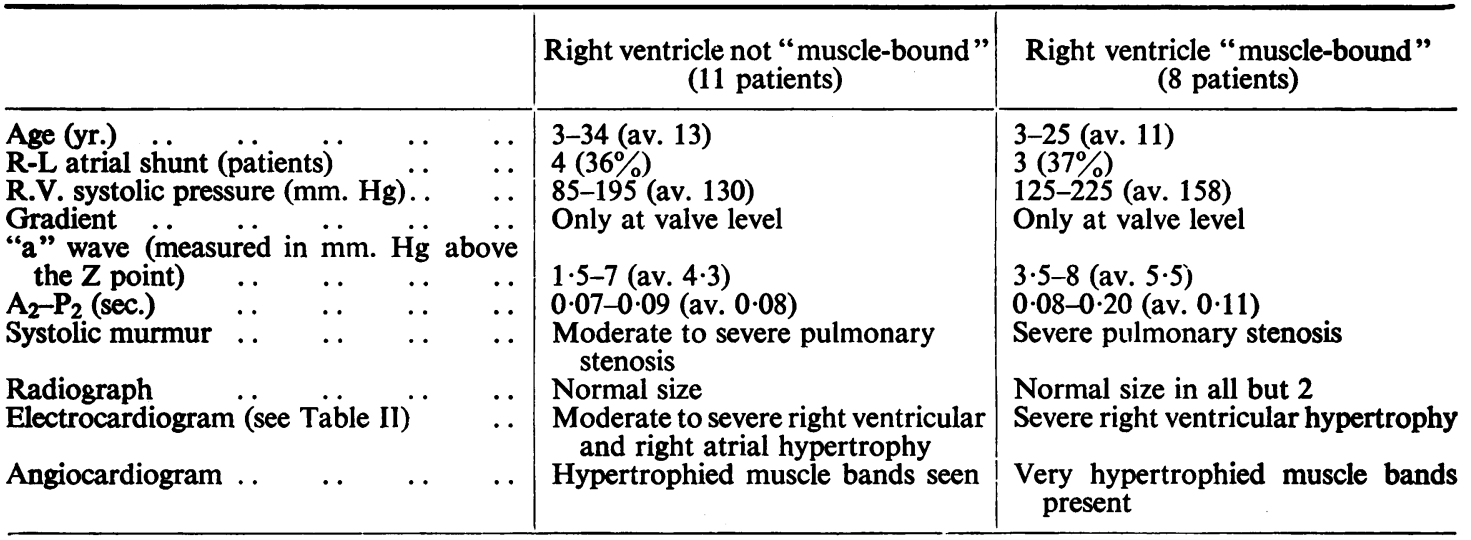

New Signs

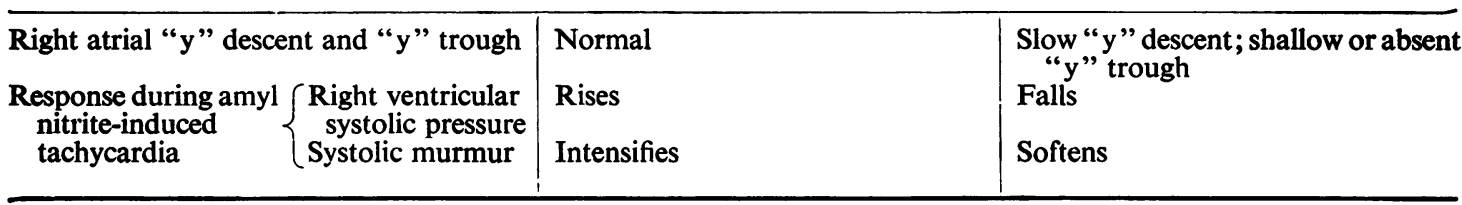

Group B. There were 8 patients in whom the right ventricle was considered to be "muscle-bound" because of the excessive degree of outflow tract hypertrophy and the failure of the right ventricular systolic pressure to fall below $100 \mathrm{~mm}$. $\mathrm{Hg}$ immediately after the valvotomy. In 7, the surgeon considered it necessary to resect the infundibular muscular stenosis; in the remaining patient severe infundibular stenosis was allowed to persist. In none was stenosis of the valve ring present.

The various clinical, electrocardiographic, phonocardiographic, radiological, and hæmodynamic parameters were analysed, and the data in each group were compared (Table I). Special attention was devoted to the right atrial pressure pulse and externally recorded jugular venous tracings, and to the response of the systolic murmur and right ventricular systolic pressure to an inhalation of amyl nitrite. The amyl nitrite studies were frequently repeated before and after surgery.

Phonocardiographic recordings of the systolic murmur were made at fast speed $(75-80 \mathrm{~mm}$./sec.) during held expiration before, during, and after an inhalation of amyl nitrite, using the technique previously described (Vogelpoel et al., 1959). These were repeated with simultaneously recorded right ventricular and sometimes right atrial pressure tracings during cardiac catheterization. The recumbent posture was always used.

The change in intensity of the systolic murmur was obtained from the high frequency phonocardiographic recording. The attenuation chosen for the control tracing was kept constant and recordings were always made during held expiration. Because there was no sound calibrating system in the N.E.P. apparatus used, the following method of measurement was devised to obtain a plot of the change in intensity of the systolic murmur against time, before and after amyl nitrite inhalation. The amplitude of the peak deflections in each systole was measured in millimeters, and the mean figure was obtained for each fast strip panel (usually 6-10 systoles). Measurements of the murmur intensity were thus obtained before and at known times after the inhalation. The change in intensity was then expressed as a figure above or below the control amplitude which was expressed as zero. Since the data have no quantitative meaning the graphs so constructed are only intended to show the trend of change in intensity (see Fig. 1).

All phonocardiographic tracings were analysed in this fashion. Generally the changes recorded in the clinic room were very similar to those found during cardiac catheterization. However, whenever possible, the phonocardiogram selected for analysis was that recorded at cardiac catheterization, so that the changes 
A
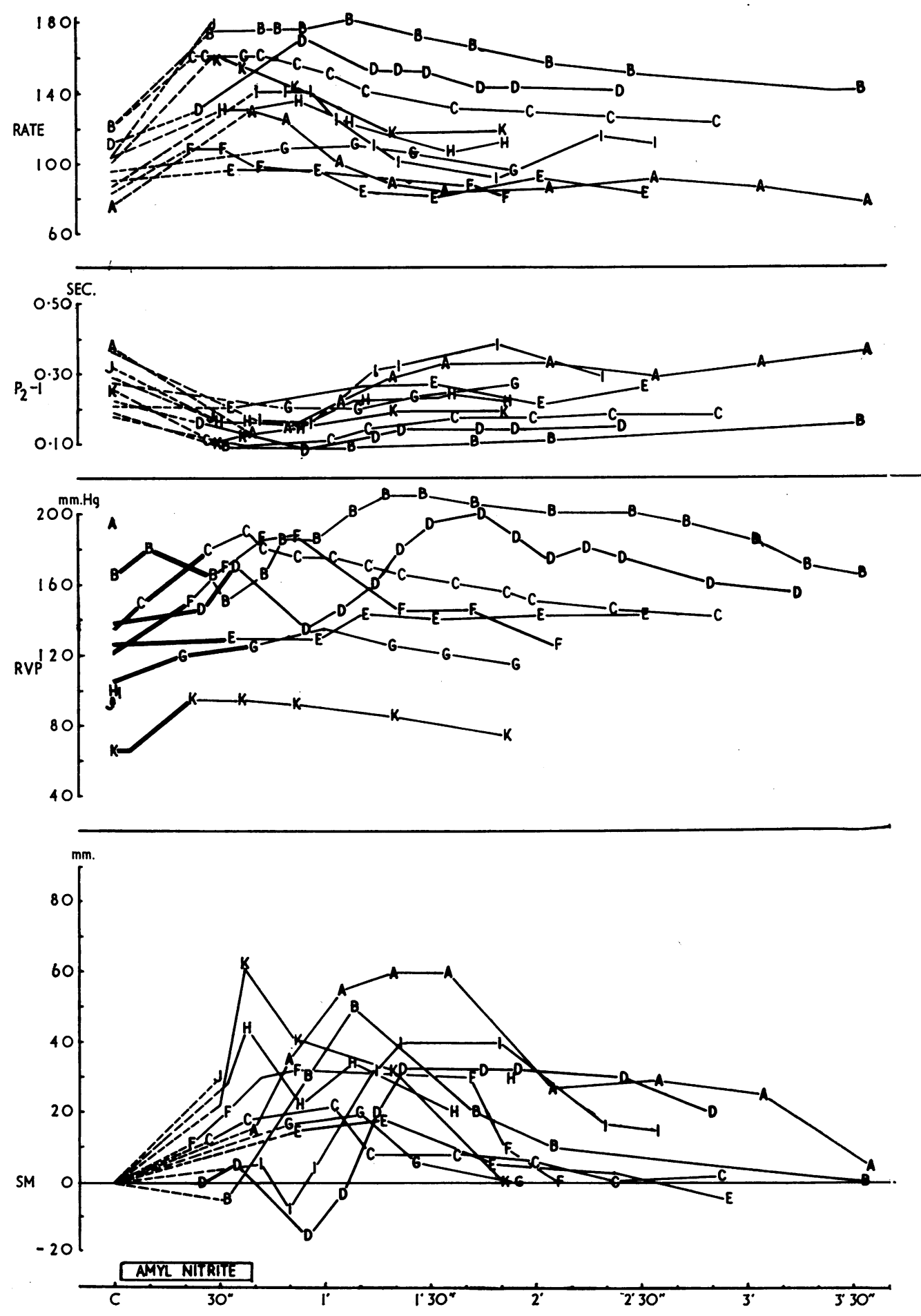

Fig. 1.-A and $B$ : Comparison of the effect of amyl nitrite on the heart rate, the diastolic filling time $\left(P_{2}-1\right.$ interval), the right ventricular systolic pressure and the intensity of the systolic murmur. The data on each patient (stated alphabetically in Group A and numerically in Group B) were simultaneously recorded. The duration of the inhalation is indicated by heavy black lines in RVP or by broken lines in the other panels. During the tachycardia phase the right ventricle, when not "muscle-bound", was able to increase its output, as assumed from the increase in 

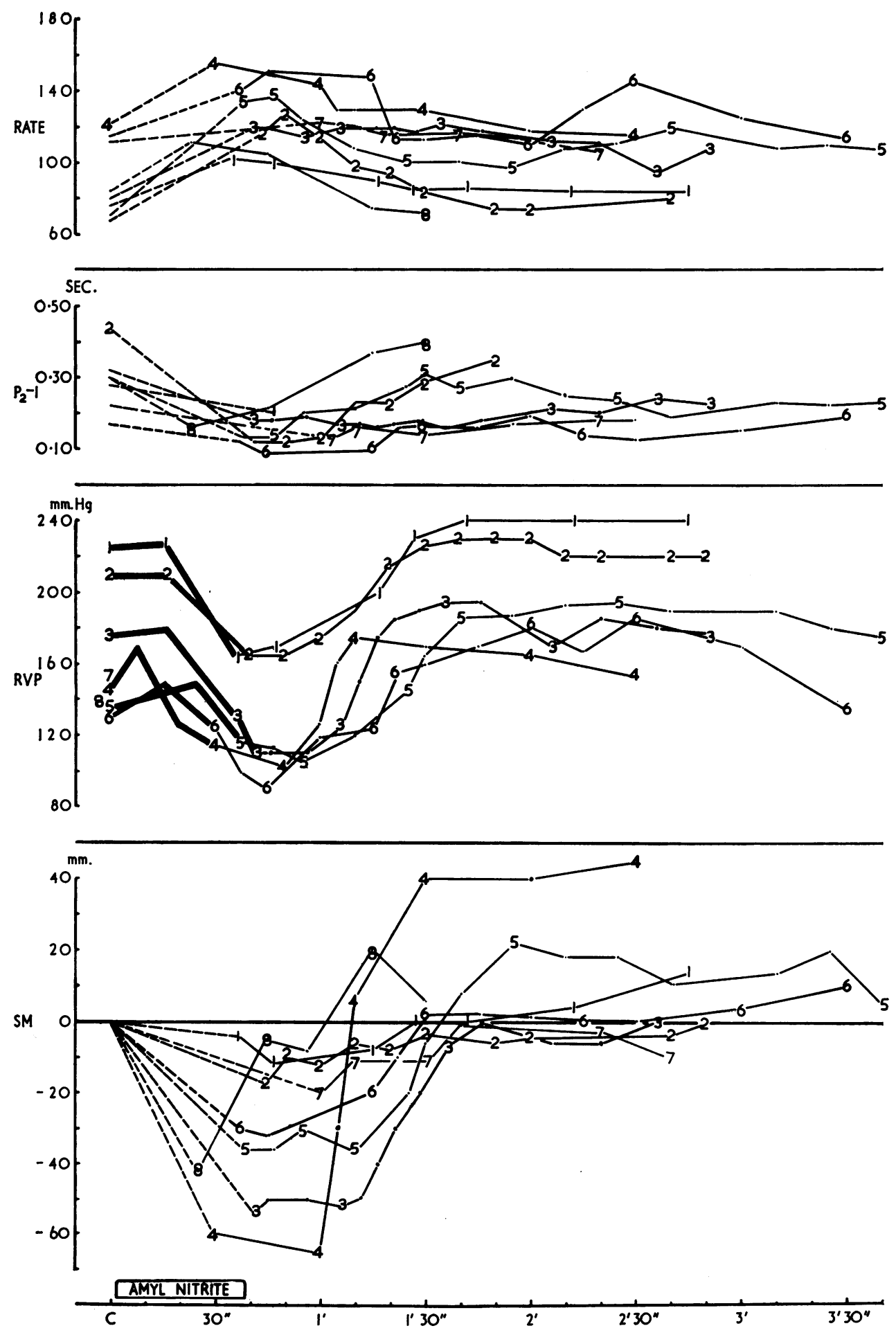

systolic pressure and murmur intensity, whereas both the pressure and murmur fell when the ventricle was "muscle-bound". Note that the diastolic filling time was not more reduced by tachycardia in the "musclebound" group. Once deceleration occurred the pressure and murmur rapidly recovered and often exceeded the control level. 


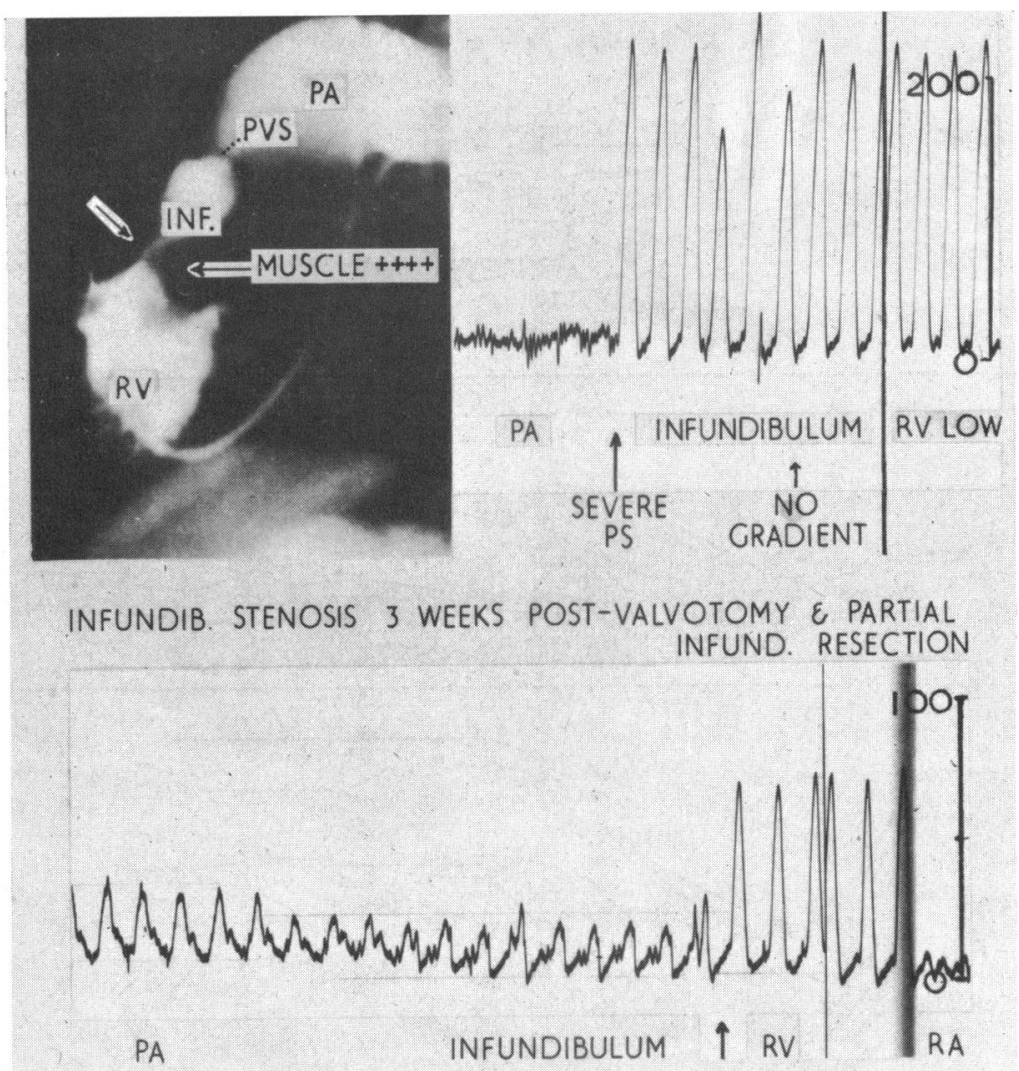

FIG. 2.-P.D., a boy, aged 14 with severe pulmonary stenosis and a "muscle-bound" right ventricle. Before operation the pressure withdrawal recording showed severe stenosis at valve level but no additional gradient in the outflow tract, despite the presence of gross hypertrophy almost shutting off the infundibulum during systole in the selective angiogram. The right atrial tracing (Fig. 3E) and the response to amyl nitrite (Fig. 1B (Case 2) and 10C) indicated a "muscle-bound" right ventricle. Severe infundibular stenosis developed immediately after valvotomy and a modest infundibular muscle resection was performed. At recatheterization three weeks later, moderate infundibular stenosis was demonstrated, the low right ventricular pressure being $75 / 10 \mathrm{~mm}$. $\mathrm{Hg}$ (bottom panel). Recatheterization one year later showed further involution (infundibulum 24/8, low RV $42 / 7 \mathrm{~mm}$. $\mathrm{Hg}$ ).

in the murmur could be correlated with the simultaneously recorded right ventricular systolic pressure, heart rate, and right ventricular diastolic period. The latter was obtained from the time interval between the pulmonary second sound, when recordable, and the first heart sound, and termed the $\mathrm{P}_{2}-1$ interval.

Phonocardiogram studies with or without right ventricular pressure recordings were repeated about three weeks after surgery and again one year later.

Rapid bi-plane selective right ventricular angiograms were done in patients to confirm the diagnosis and to assess the degree of right ventricular hypertrophy. Dye dilution studies were included in the diagnostic work-up, to exclude a right-to-left shunt at ventricular level. When a right-to-left shunt was demonstrated at atrial level, the curve was repeated during the peak action of amyl nitrite, using a method previously described (Vogelpoel et al., 1962).

\section{RESULTS}

The marked overlap of the various diagnostic parameters in the two groups, summarized in Table I, shows the difficulty in the pre-operative recognition of the degree of right ventricular muscular hypertrophy in a given case of pulmonary valve stenosis. Thus, the age of the patient, which expresses the duration of the disease, the presence of a right-to-left interatrial shunt, the 
TABLE II

SEVERITy of Right Ventricular HyPertrophy on Electrocardiogram

\begin{tabular}{ll|l|l|l|l|l}
\hline & \multicolumn{6}{|c}{ Grade of severity } \\
\cline { 3 - 7 } & & 0 & 1 & 2 & 3 & 4 \\
\hline $\begin{array}{lllll}\text { Right ventricle not "muscle-bound" } \\
\text { Right ventricle "muscle-bound" }\end{array}$ & $\ldots$ & 0 & 3 & 6 & 2 & 0 \\
\hline
\end{tabular}

height of the "a" wave, the length of the systolic murmur, the width of splitting of the second sound, and the radiological appearances usually failed to distinguish between the two groups.

The electrocardiogram had some value in that extreme right ventricular hypertrophy (Grade 4) was found only in the "muscle-bound" group, but since the majority of patients in both groups showed Grade 2-4 severity, the role of the cardiogram in the diagnosis had limited value (Table II).

Whereas excessive hypertrophy was encountered only in patients with severe valve stenosis (right ventricular pressure above $120 \mathrm{~mm}$. $\mathrm{Hg}$ ), the mere finding of a high systolic pressure did not inevitably mean a "muscle-bound" right ventricle. The average right ventricular systolic pressure in the non "muscle-bound" group was 130 and the highest figure was $195 \mathrm{~mm}$. $\mathrm{Hg}$, compared with the "muscle-bound" group where there was an average of 158 and a highest figure of $225 \mathrm{~mm}$. Hg.

Pressure withdrawal tracings from the pulmonary artery to the right ventricle invariably failed to detect any additional gradient in the infundibulum (Fig. 2). However, following complete valvotomy the shift in gradient to the infundibular region could be demonstrated (Fig. 2).

Rapid bi-plane selective right ventricular angiograms proved helpful in demonstrating the hypertrophied muscle bands and their behaviour during the cardiac cycle (Fig. 2). Hypertrophied bands were impressive in both groups but we were unable to define angiographic criteria, by which a "muscle-bound" ventricle could be recognized.

The following observations appeared to distinguish between the two groups pre-operatively (Table I).

The Right Atrial Pressure Pulse. Whereas the height of the "a" wave did not differentiate the two groups (Table I), the appearance of the " $y$ " descent and " $y$ " trough was very different, provided tachycardia did not interfere with their inscription. In those without a "muscle-bound" right ventricle, the " $y$ " descent and trough appeared normally rapid and deep (Fig. 3A). By contrast when the right ventricle was excessively hypertrophied, the " $y$ " descent was very slow and the trough either shallow or absent (Fig. 3B-E) suggesting gross impedance to diastolic filling of the right ventricle. The tracing closely resembled that of tricuspid stenosis, yet none had this condition. This abnormality is confined to the right heart, as shown in Fig. 3C, where the " $y$ " descent and trough were grossly abnormal in the right atrium but normal in the left, entered via a patent foramen ovale.

In Fig. 3D the right atrial tracing showed a deep " $x$ " descent but no " $y$ " descent during early right ventricular diastole. However, at recatheterization one year after pulmonary valvotomy and infundibular muscular resection, the right atrial tracing showed a normal appearance with a rapid " $y$ " descent and deep " $y$ " trough. The right ventricular pressure had fallen to normal and the systolic murmur was now that of mild pulmonary stenosis.

In Fig. 3E the " $y$ " descent was extremely slow despite the bradycardia present. However, three weeks after complete valvotomy and partial infundibular resection there was a remarkable transformation in that the " $y$ " descent was rapid and the trough deep, but followed by a rapid climb to the " $z$ " level before the ensuing " $a$ " wave. The change in the right atrial wave form suggested partial improvement in right ventricular diastolic filling, the configuration now resembling that described by Johnson (1962).

External qualitative recordings from the internal jugular vein showed essentially the same 2D 


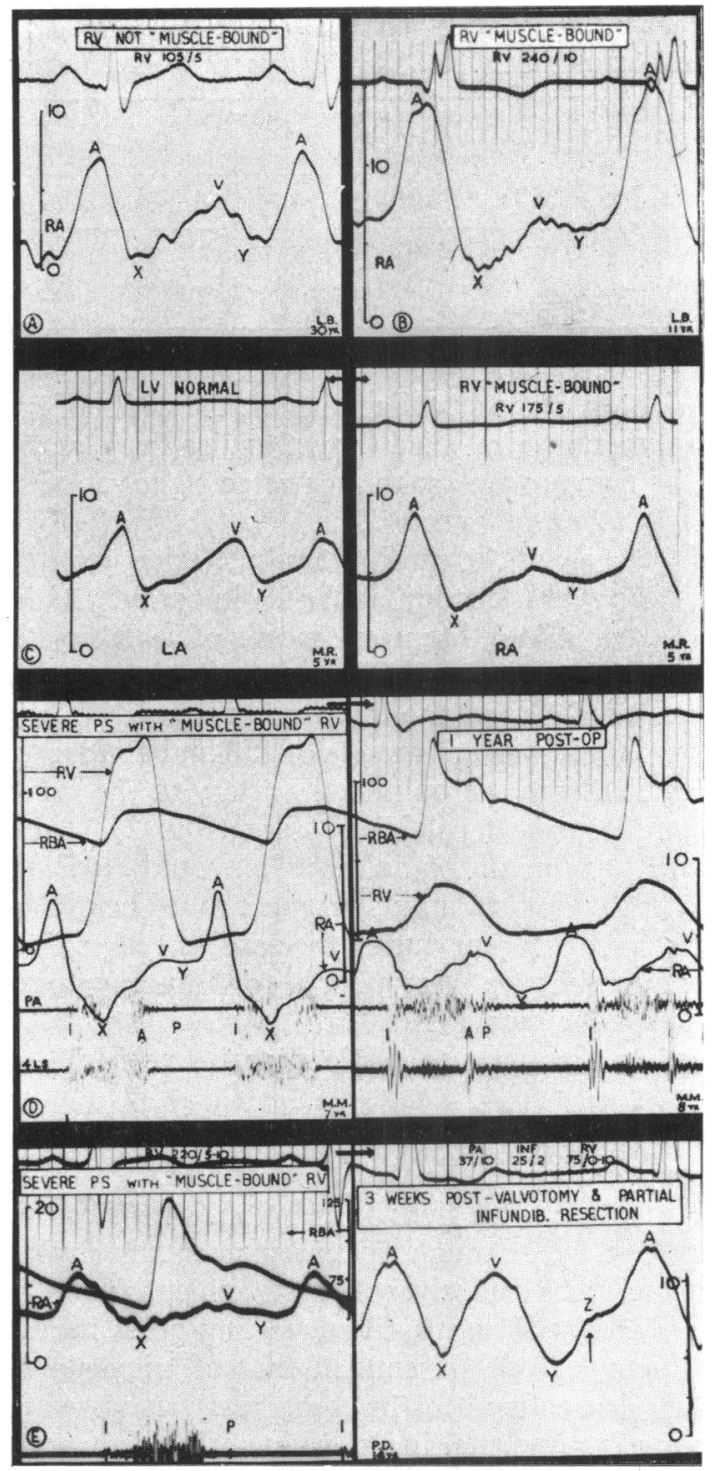

FIG. 3.-The right atrial pulse in pulmonary stenosis. The feature that distinguished the "muscle-bound" right ventricle was the abnormally slow " $y$ " descent and shallow or absent " $y$ " trough (B to E). This pattern was not found when the right ventricle was not "muscle-bound" (A), or in the left atrium (C), and it disappeared after successful surgery (D), and changed after partially successful surgery (E). See text.

features and were useful in differentiating the two groups, provided that tachycardia was absent and the tracings were technically good (Fig. 4).

Response of Systolic Murmur and Right Ventricular Systolic Pressure to Amyl Nitrite. The effect of amyl nitrite inhalation on the right ventricular systolic pressure, on the intensity of the systolic murmur, on the heart rate, and on the right ventricular diastolic filling period has been plotted against time in Fig. 1. The data for each patient were obtained simultaneously, though in six subjects right ventricular pressure recordings were not made following amyl nitrite. 


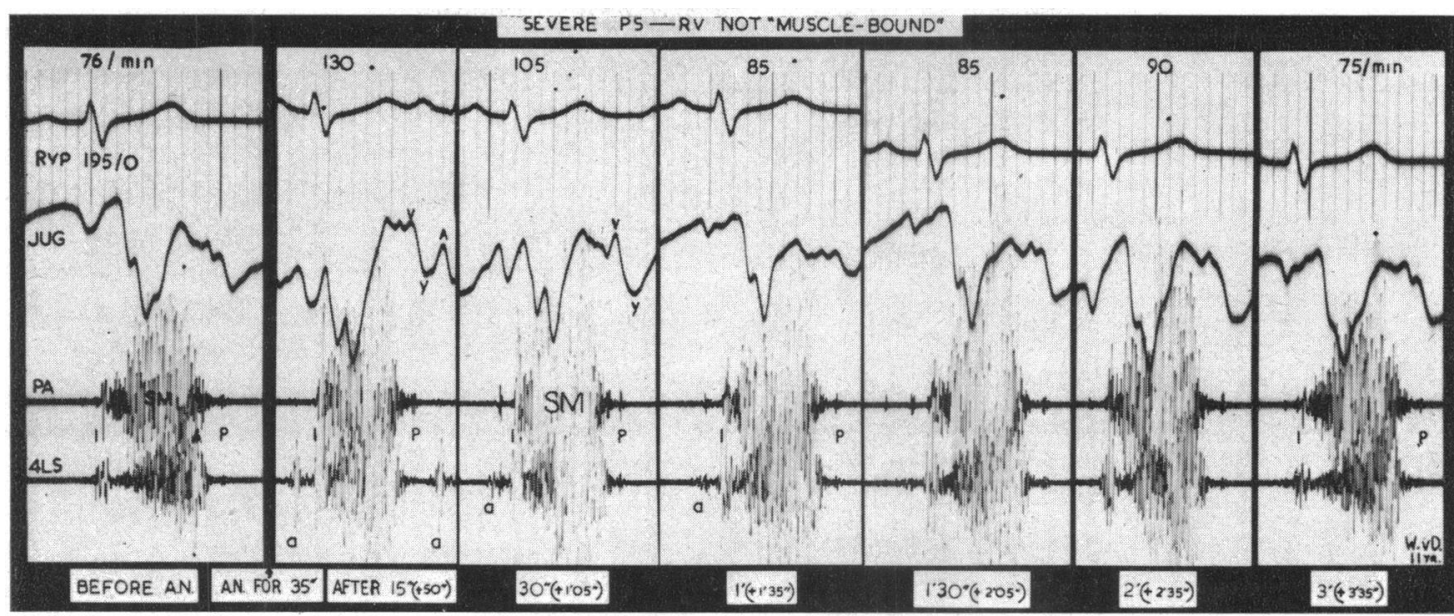

FIG. 4.-Case A, W.v.D., a boy, aged 11, was a good candidate for having a "muscle-bound" right ventricle, having a very high right ventricular pressure of $195 / 0 \mathrm{~mm}$. $\mathrm{Hg}$. However, the normal " $\mathrm{y}$ " descent in the jugular tracing, which remained evident even during the tachycardia phase, indicated a normal diastolic filling resistance. This was also suggested by the intensification of the systolic murmur during the tachycardia phase, indicating that the right ventricle was able to increase its ejection rate despite the reduced filling period. Disproportionate right ventricular hypertrophy was not found at operation, the right ventricular pressure dropping to $30 / 2 \mathrm{~mm}$. $\mathrm{Hg}$, immediately after the valvotomy.

In all patients amyl nitrite produced a tachycardia and a shortened right ventricular filling period, which reached a peak at about 30 seconds from the start of the inhalation, remained at this level for about 20-30 seconds, and then slowly returned to the control level, which was reached in about 3 to $3 \frac{1}{2}$ minutes. Although faster rates were attained in the group without "muscle-bound" right ventricles, there was very little difference in the degree of curtailment of the right ventricular diastolic period. This was attributed to the longer systolic period with greater delay in $\mathbf{P}_{2}$ in the group with "muscle-bound" right ventricles (see $\mathrm{A}_{2}-\mathrm{P}_{2}$ times in Table I). However, despite the similar degree of reduction in the right ventricular diastolic filling period, the right ventricular systolic pressure and systolic murmur intensity behaved very differently in the two groups.

In the subjects whose right ventricles were not "muscle-bound" (Fig. 1A), the right ventricular systolic pressure rose and the systolic murmur intensified during the acceleration and peak tachycardia phase following amyl nitrite (see also Fig. 4 and 7A). In 3 patients (D, B, and I) just before the peak tachycardia phase was reached, there was slight softening of the murmur and some reduction in the right ventricular systolic pressure, following an initial rise in B and D. However, once the peak tachycardia had been reached, a significant rise in right ventricular pressure and murmur intensity occurred, and these continued to rise during the gradual deceleration in rate. Patients $B$ and $D$ achieved the fastest rates and greatest shortening of the right ventricular diastolic filling period. Both were partially "muscle-bound" in that the right ventricular systolic pressures fell from 165 to 65 in B and 135 to $75 \mathrm{~mm}$. $\mathrm{Hg}$ in D, respectively, after complete valvotomy, but both showed complete involution on recatheterization one year later.

In the subjects whose ventricles were "muscle-bound" (Fig. 1B), the right ventricular systolic pressure fell, and the systolic murmur softened greatly during the acceleration phase, reaching a nadir during the peak tachycardia phase and then rapidly recovered as the pulse rate commenced decelerating (see also Fig. 5, 6, 7B). Return to the control level usually occurred between 1 and $1 \frac{1}{2}$ minutes after the inhalation, and thereafter the systolic pressure continued to rise above the control level in all 6 subjects whose pressures were measured. However, in only 5 did the systolic murmur exceed the control level. During the inhalation, the right ventricular systolic pressure, recorded at slow rate (Fig. 1B, heavy black lines), rose during the first 10-15 seconds in 4, but at a 


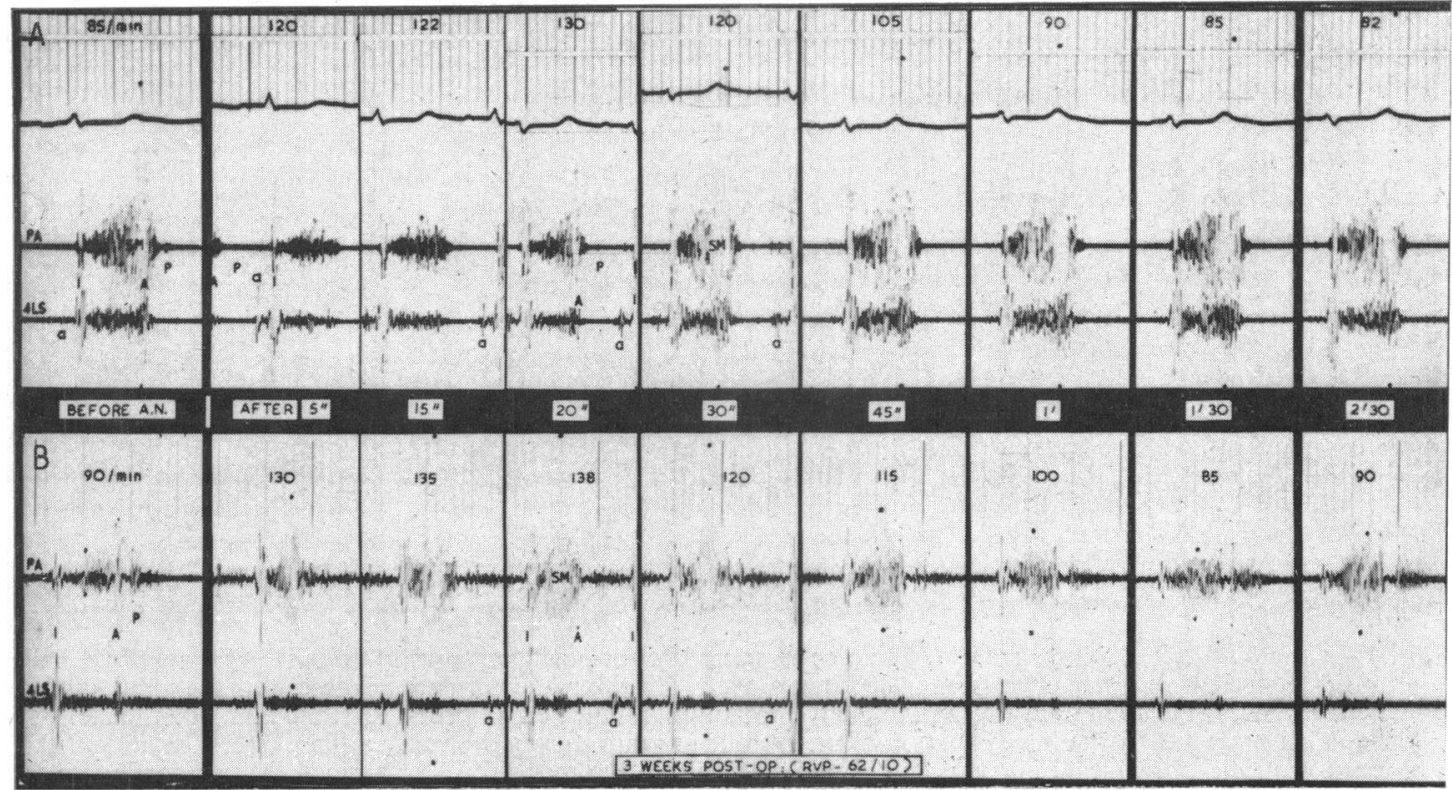

Fig. 5.-Case 6, F.W., a boy, aged 10, had severe pulmonary stenosis with a right ventricular pressure of $125 / 10 \mathrm{~mm}$. $\mathrm{Hg}$. The systolic murmur before operation (A) softened greatly but did not shorten during moderate amyl nitrite-induced tachycardia following a 30 -second inhalation. Thereafter, with deceleration the murmur rapidly recovered and exceeded the control level. Severe secondary infundibular stenosis developed after valvotomy, requiring infundibular resection. Three weeks later (B) the shorter and softer murmur intensified greatly during the tachycardia phase, thereafter decreasing rapidly with deceleration. The prompt normalization of the right ventricular response suggested that the impedance to right ventricular filling had been corrected by the operation.

certain critical point, presumably determined by the heart rate and shortening of the right ventricular diastolic filling period, the pressure suddenly dropped and then fell progressively, as the heart rate continued to rise (see also Fig. 6). Because recordings were made at slow speed during the inhalation, the change in rate, $\mathrm{P}_{2}-1$ time, and murmur intensity at this stage could not be measured, being shown as broken lines on the graph.

Illustrative cases are described briefly in the legends of Fig. 2 and 4-7 where the case number or letter corresponds to that used in Fig. 1.

\section{Discussion}

An inhalation of amyl nitrite is a simple and effective method of provoking a greatly increased ventricular output. This is achieved mainly by an increase in heart rate. Within 30 seconds, the ejection rate of both ventricles rises rapidly, provided that a brisk systemic pressure drop and tachycardia have occurred, and that myocardial failure is absent. When given in the recumbent posture there is good evidence of increased venous return to the right ventricle following on the severe reduction in systemic resistance and despite the likelihood of venous pooling (Honig, Tenney, and Gabel, 1960; Beck et al., 1961).

The moment to moment changes in venous return and right ventricular output, however, are at best difficult to quantitate during the short-lived, unsteady state induced by this drug. With the techniques available to us these measurements could not be made. Fortunately in pulmonary stenosis with intact ventricular septum, the right ventricular systolic pressure and the pulmonary ejection systolic murmur are two easily measured parameters, which provide useful qualitative information concerning the performance of the right ventricle. A change in right ventricular pressure must reflect a change in right ventricular output, while a change in murmur intensity 


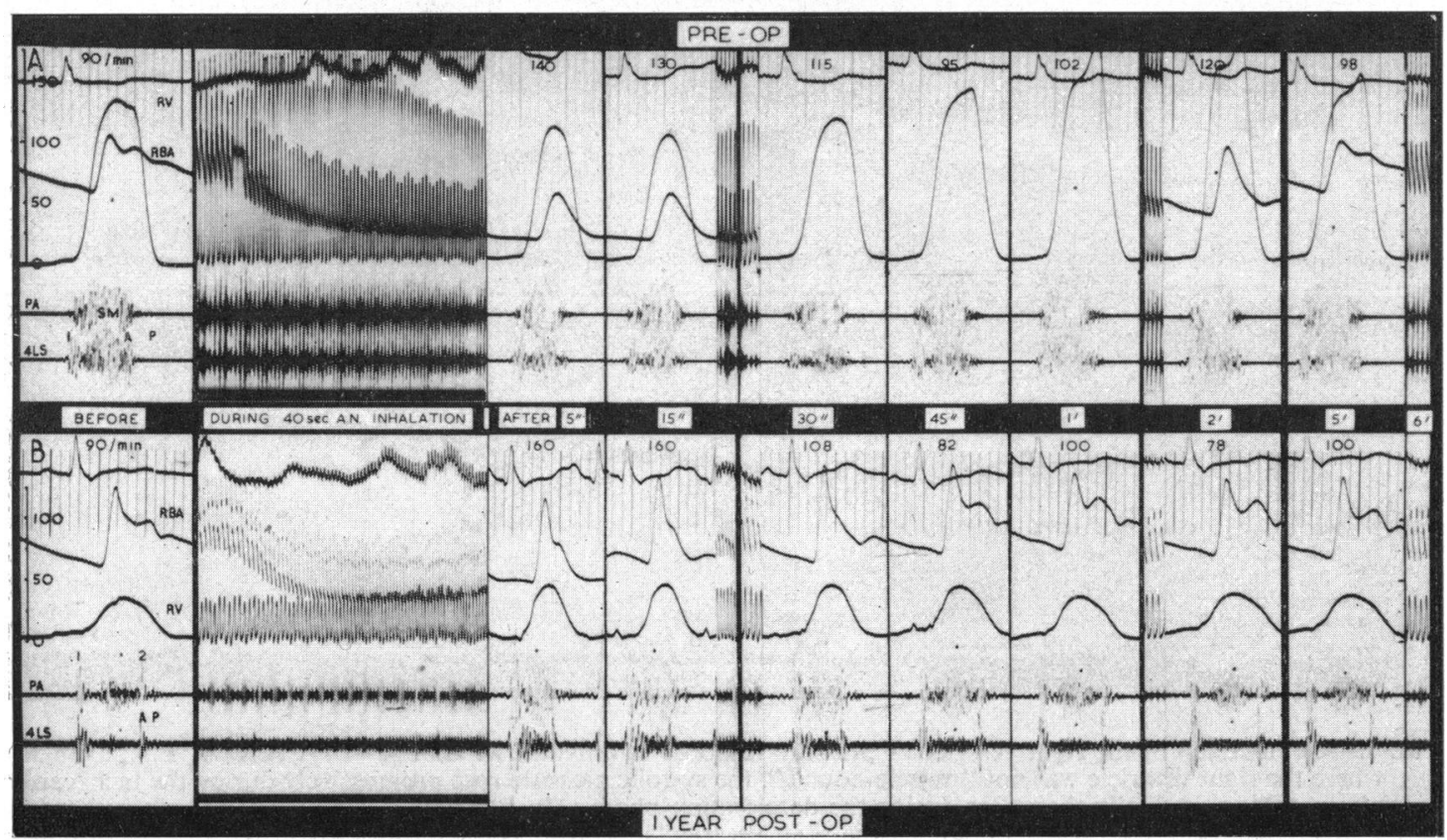

Fig. 6.-Case 5, M.M., a boy, aged 11, had severe pulmonary stenosis with the right ventricular systolic pressure exceeding the systemic. Before surgery (A) the right ventricular systolic pressure and the intensity of the systolic murmur decreased during the tachycardia phase but rapidly recovered and exceeded the control level during the deceleration phase. Of special interest was the initial rise in right ventricular pressure which followed soon after the onset of systemic hypotension and tachycardia. However, as the tachycardia neared its maximum, the right ventricular systolic pressure dropped suddenly and progressively, suggesting a temporary fall in right ventricular output. The right ventricle was "muscle-bound" and an infundibular resection was required. The study was repeated one year later (B). The right ventricle was now able to increase its pressure and ejection murmur despite greater tachycardia, and there was a quick return to control level during the deceleration phase. Recordings made at 2.5 and $75 \mathrm{~mm}$./sec.

reflects a change in the velocity of ejection through the stenosed orifice. On the assumption, therefore, that a rise in both pressure and murmur intensity indicates a rise in right ventricular ejection rate, and vice versa, the data obtained in this study can be interpreted as follows.

In pulmonary stenosis, when the right ventricle is not excessively hypertrophied, it can increase its pressure and ejection rate during the tachycardia phase induced by amyl nitrite. However, when excessively hypertrophied ("muscle-bound"), the right ventricle performs much less efficiently. Even for modest increases in heart rate, the marked drop in pressure and murmur intensity suggests a significantly reduced ejection rate. Once deceleration commences, the right ventricle rapidly recovers and is now able to increase its pressure and ejection rate. Such a ventricle is evidently capable of efficient function at normal heart rates but is severely handicapped by a fast rate. Correction of the stenosis and removal of excess muscle in the right ventricle promptly restores its ability to increase its ejection velocity during severe amyl nitrite-induced tachycardia (Fig. 5 and 6).

In order to explain why the right ventricular output (stroke volume $\times$ heart rate) should drop during the tachycardia phase when the ventricle is "muscle-bound", it is necessary to examine the factors that determine the stroke volume. These are the systolic contractility and diastolic filling functions of the right ventricle (Fig. 8).

1. Systolic Contractility. Defective contractility cannot be an important factor. Cardiac failure was always absent and no patient was found with a dilated right ventricle at surgery. In fact it is our experience that when cardiac failure is present in pulmonary stenosis, the right ven- 


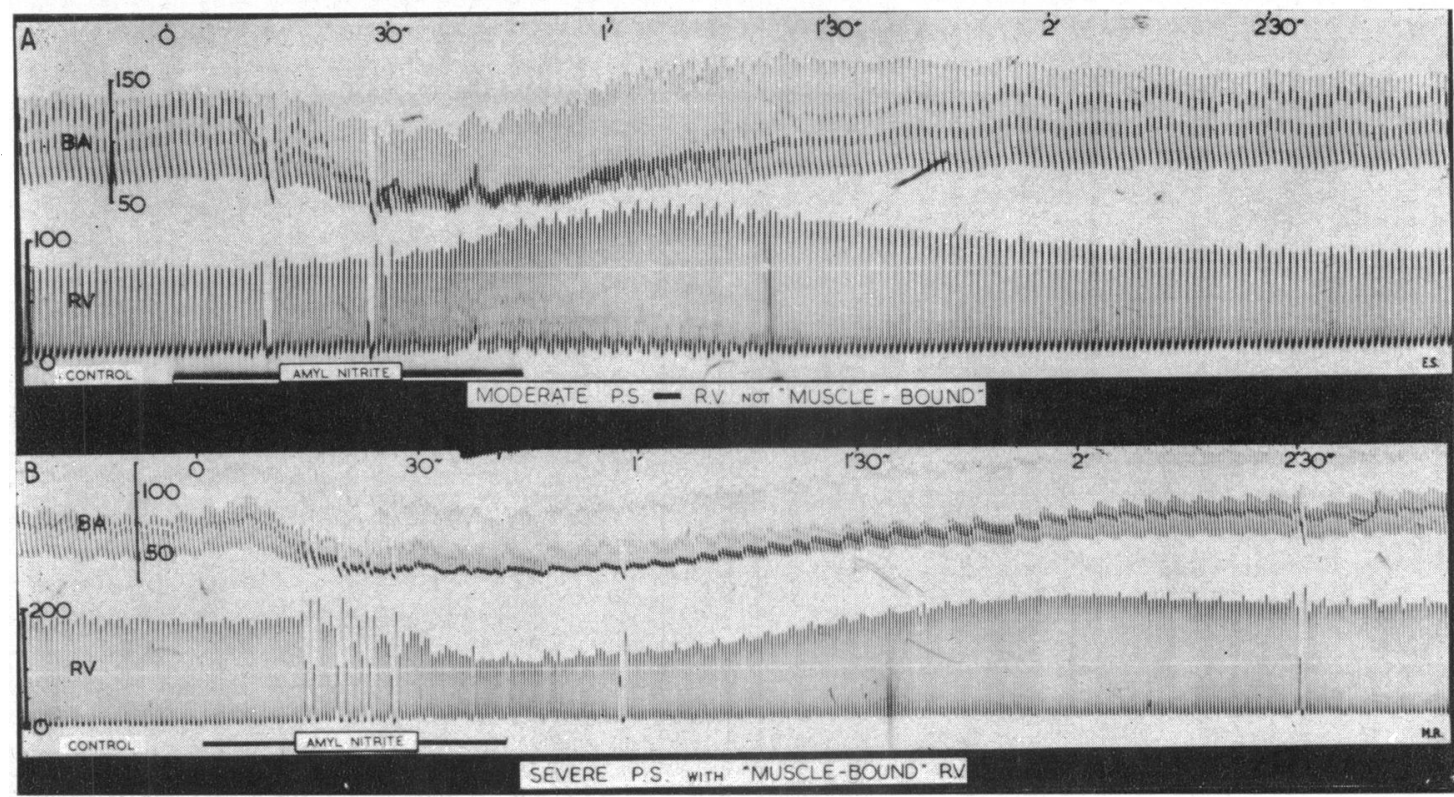

FIG. 7.-Comparison of the right ventricular pressure response to amyl nitrite action. In A (Case J, E.S., aged 36), where the right ventricle was not "muscle-bound", the systolic pressure rose progressively during the tachycardia phase, falling gradually thereafter during the deceleration phase. In B (Case 3, M.R., aged 5), where the right ventricle was severely "muscle-bound", the right ventricular systolic pressure dropped progressively during the tachycardia phase and recovered slowly with deceleration and eventually slightly exceeded the control level. Note the similar effect of amyl nitrite on the brachial artery pressure (BA) in each subject.
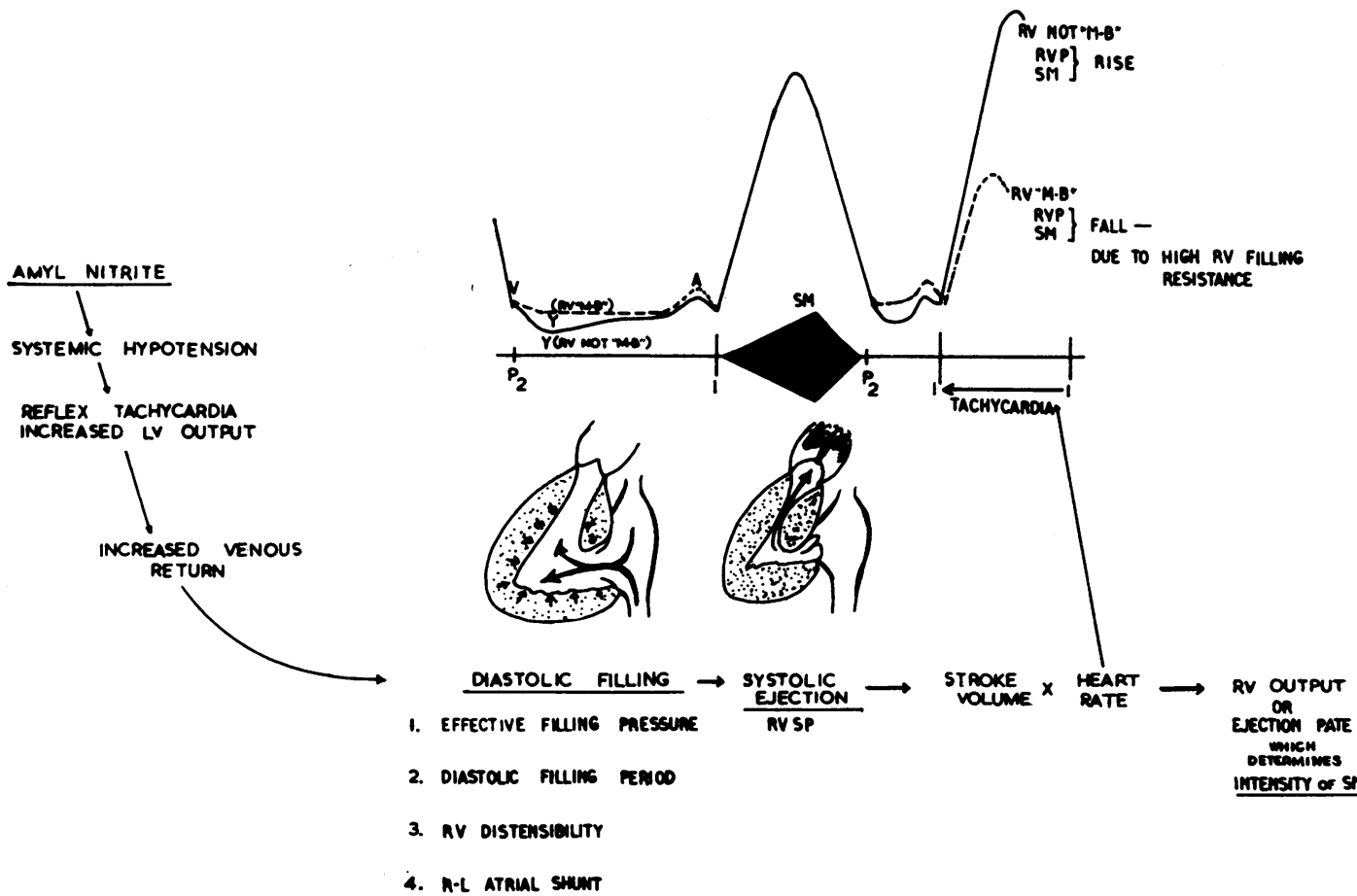

Fig. 8.-Diagram depicting the factors that determine right ventricular performance during amyl nitrite-induced tachycardia in pulmonary stenosis with intact ventricular septum. Reduction of the $P_{2}-1$ interval to the same degree by tachycardia is associated with increased right ventricular systolic pressure and murmur when the right ventricle is not "muscle-bound" but reduced performance when the ventricle is "muscle-bound". This difference is attributed to the greater diastolic filling resistance when the ventricle is excessively hypertrophied, which is also shown by the abnormally slow "y" descent and shallow "y" trough in the right atrial pulse. See text. 


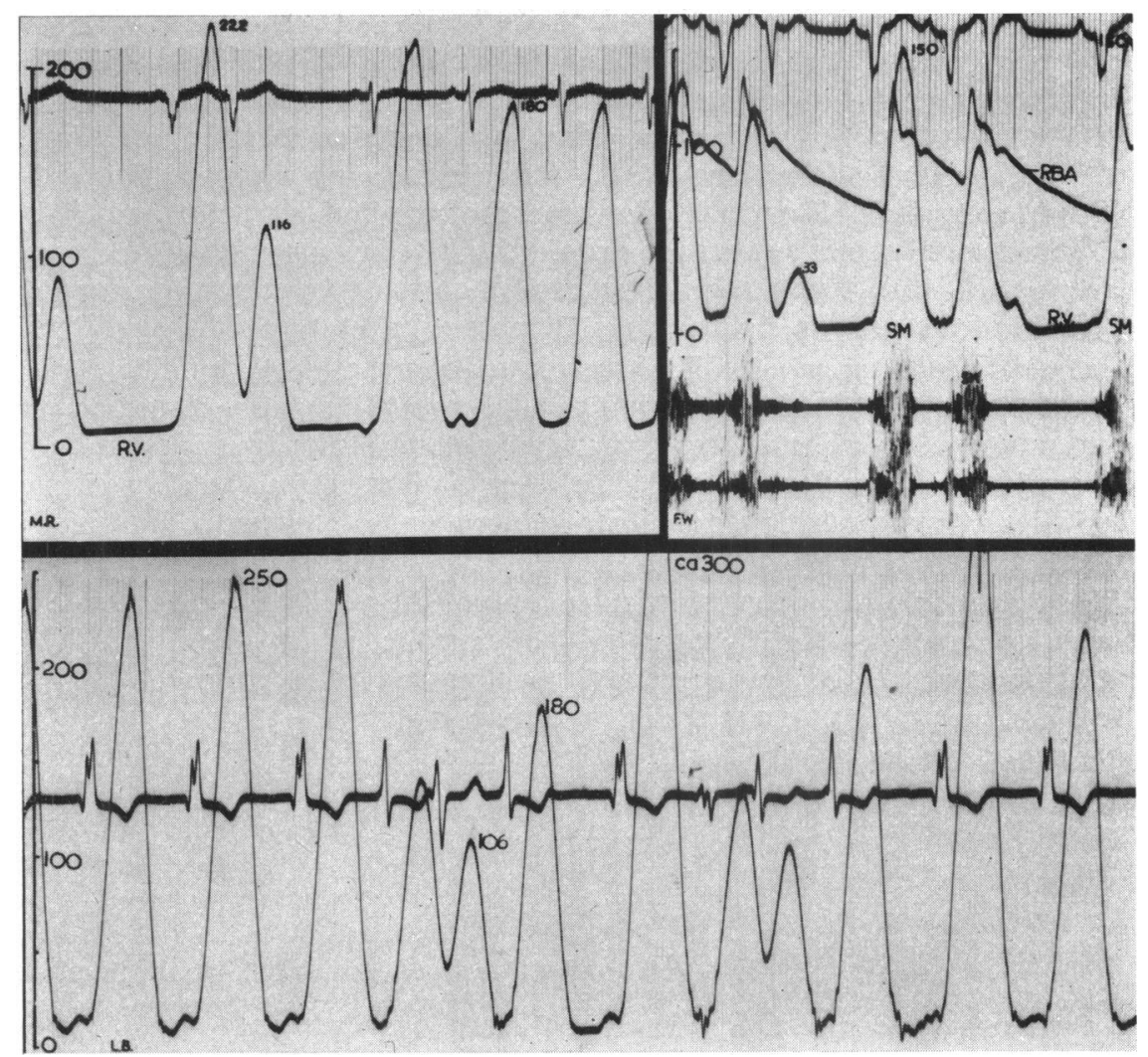

FIG. 9.-The effect of varying diastolic cycle lengths on the right ventricular systolic pressure in 3 patients with severe pulmonary stenosis and "muscle-bound" right ventricles. After long pauses that enable greater diastolic filling, the systolic pressure and ejection systolic murmur far exceed the control level. Following short pauses there is a striking fall in systolic pressure and sometimes a significant rise in diastolic pressure, the result of insufficient relaxation time.

tricular systolic pressure and murmur intensity change very little after amyl nitrite. That contractility was unimpaired could be inferred from the very high pressures generated by the right ventricle after a compensatory pause following a premature beat (Fig. 9). Three examples are shown illustrating that when a "muscle-bound" right ventricle is given enough time to fill, the systolic pressure far exceeds the basal level. Conversely, when made to contract prematurely the systolic pressure generated is remarkably low, even for beats that are ordinarily very little premature. These simple observations suggest that the "muscle-bound" right ventricle in pulmonary stenosis is extremely powerful and that during premature beats and presumably during sinus tachycardia after amyl nitrite, the marked drop in systolic pressure is not due to defective contractility.

2. Diastolic Filling. This is dependent on (a) the effective filling pressure. In 3 patients without "muscle-bound" right ventricles, the right atrial pressure failed to change during the tachycardia phase, and in 1 patient with a "muscle-bound" ventricle this pressure rose slightly. However, in the absence of flow data, the change in effective filling pressure of the right ventricle could not be interpreted from right atrial pressure changes. None the less the prompt and progressive rise in right ventricular systolic pressure in mild and moderate pulmonary stenosis is good indirect evidence that amyl nitrite inhalation in the recumbent position produces an increased venous return and effective filling pressure (Fig. 7A). It is inconceivable that the systemic effects of amyl nitrite would be any different when the right ventricle is "muscle-bound". Rather it seems 
that the venous return increases, irrespective of the degree of right ventricular hypertrophy, but that the excessively hypertrophied right ventricle cannot increase its filling in the presence of tachycardia.

(b) The influence of a right-to-left atrial shunt. In 3 patients with "muscle-bound" right ventricles and 4 without, small right-to-left atrial shunts were shown to increase following amyl nitrite. This could have decompressed the right atrium, thereby lessening right ventricular filling and its response (Fig. 1A, cases B, E, F and I). However, 5 of the patients with "muscle-bound" right ventricles had intact atrial septa, so that the drop in right ventricular output in these patients was clearly not due to right-to-left shunting. Thus, an interatrial communication cannot be invoked as a primary cause of the drop in right ventricular output in the "muscle-bound" right ventricle.

(c) Reduced diastolic filling period. Tachycardia reduces the diastolic filling period to a much greater extent than the systolic. Diastolic filling in the normal human ventricle becomes critically reduced at rates above 180 a minute, resulting in underfilling of the ventricles and a fall in stroke volume (Wiggers, 1949). This figure is probably lower in older persons and higher in children, but since the peak rates attained during amyl nitrite-induced sinus tachycardia are usually well below the critical range, a significant rise in cardiac output occurs (Beck et al., 1961).

In pulmonary stenosis with intact ventricular septum the prolonged right ventricular systolic period encroaches severely on the right ventricular diastolic period (Fig. 10). The greater the prolongation of right ventricular systole (delay in pulmonary valve closure), the greater this encroachment. Hence it will be apparent that in severe pulmonary stenosis the right ventricular diastolic filling period will be reduced to critical levels at rates much lower than in the normal right ventricle or in the left ventricle of the same heart.*

In order to determine whether the reduced right ventricular output in the "muscle-bound" subjects was simply the result of their slightly longer systolic periods, the diastolic periods $\left(\mathrm{P}_{2}-1\right.$ interval) were measured during the peak tachycardia phase. In Fig. 1 and 10 it is shown that the diastolic filling time was not in fact more reduced by tachycardia in the "muscle-bound" group. Hence, some other factor must account for the fall in right ventricular output in this group.

(d) Right ventricular diastolic filling resistance. There are several good reasons for attributing the difference in right ventricular performance to the difference in diastolic filling resistance. This is likely to be considerably higher in the group whose performance was abnormal, because all had "muscle-bound" right ventricles and an abnormally slow " $y$ " descent and "y" trough in the right atrial pulse. When it is appreciated how extremely thick and stiff the right ventricle can become, often with secondary fibrotic changes (Brock, 1955, 1957), ventricular distensibility must surely be grossly abnormal. The greater the impedance to filling the greater the difficulty with filling when the filling time is curtailed by tachycardia. Thus, it should not be surprising to find a severe drop in right ventricular performance during even modest tachycardia in subjects with excessive hypertrophy (Fig. 1 and 10C).

Using a carefully conducted exercise test, Johnson (1962) found an abnormally low cardiac output response to exercise in some subjects with severe pulmonary stenosis. He attributed this abnormal hæmodynamic response to an inadequate rise and sometimes a drop in stroke volume caused by relative shortening of diastole combined with increased right ventricular filling resistance. His emphasis on the latter factor is in full agreement with our argument.

In none of Johnson's abnormally responding patients did the right ventricular systolic pressure drop during the exercise test. It seems likely that exercise produces a greater venous return than amyl nitrite and a less suddenly provoked tachycardia, so that even a "muscle-bound" right ventricle should be able to increase its output, albeit inadequately. It is unlikely, therefore, that the

\footnotetext{
* No doubt several important factors come into play during tachycardia to relieve this handicap. Catecholamines may be released and act on the myocardium, shortening the systolic period by augmenting contractility, reducing myocardial viscosity, and conceivably improving myocardial distensibility (Rushmer, 1961). With the rise in right atrial pressure, tricuspid valve opening may precede pulmonary valve closure, thereby increasing the diastolic filling period (in Fig. 2D and $2 E$ the " $v$ " wave precedes $P_{2}$ without even making allowance for the delay in pressure transmission down the catheter).
} 


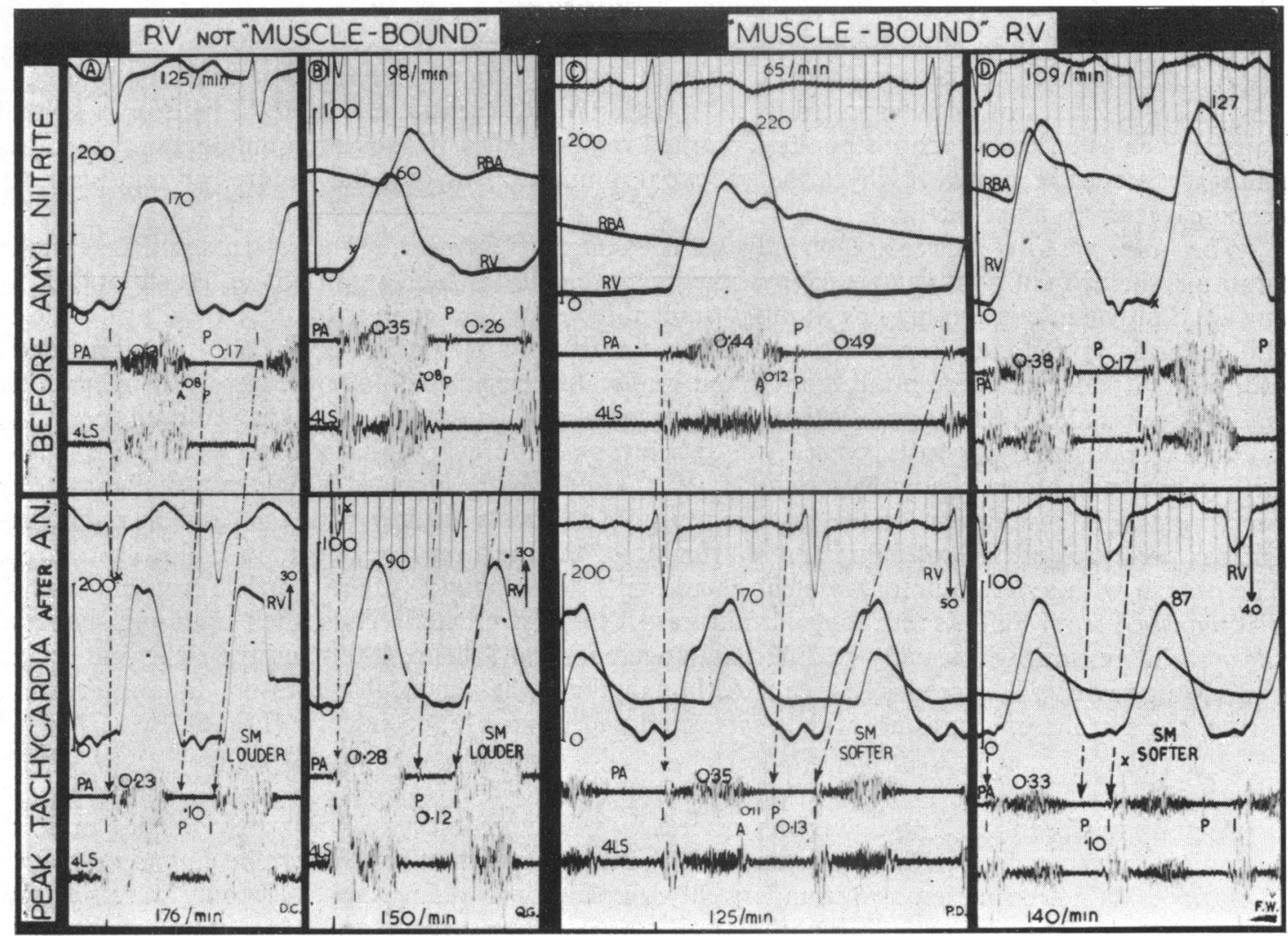

FIG. 10.-Two patients (A and B) without "muscle-bound" right ventricles are compared with two (C and D) whose ventricles were "muscle-bound". The recording during the peak tachycardia phase is mounted below the control to show the change in systolic and diastolic periods, indicated by arrows. Despite the faster rates and slightly shorter diastolic periods attained by A and B, their right ventricles were able to increase their systolic pressure and ejection murmurs greatly, whereas in $\mathbf{C}$ and $\mathbf{D}$ there was a big drop in right ventricular performance. See text.

exercise test will distinguish the two groups as readily as the amyl nitrite test. The value of amyl nitrite in exposing a "muscle-bound" ventricle lies in the fact that within 30 to 40 seconds after the start of a good inhalation, the right ventricle is compelled to increase its output by and in spite of tachycardia and without the help of as great a venous return. Ventricular distensibility is thus critically tested and indirectly reflected by the manner in which the right ventricle performs.

Practical Application of the Amyl Nitrite Test. The test is safe and can be used at the bedside or during cardiac catheterization. The simplest method is to observe the effect of a good inhalation on the right ventricular pressure recorded continuously at slow speed (Fig. 7). While obvious intensification or softening of the systolic murmur is easy to hear, it is much more difficult to be sure about lesser changes; for this reason a good phonocardiographic tracing at fast speed or a taperecording is essential in studying the effect of amyl nitrite on the systolic murmur.

If the right ventricular systolic pressure and ejection murmur increase rapidly, diastolic filling of the right ventricle is normally efficient, excessive hypertrophy will not be encountered, and serious secondary infundibular obstruction following valvotomy will not occur. Patients showing this type of response require a pulmonary valvotomy only. However, if there is a fall in right ventricular systolic pressure and a significant softening of the systolic murmur during the tachycardia phase, especially if the tachycardia is modest, then severe impedance to diastolic filling is present, implying 
an excessively hypertrophied, "muscle-bound" ventricle. Severe secondary infundibular stenosis can be anticipated, and infundibular resection may be required.

A flat response, i.e. no significant change in right ventricular pressure or murmur following amyl nitrite, must not be interpreted as a "muscle-bound" ventricle. It could be due to several causes, such as excessive venous pooling, marked right-to-left atrial shunting, moderately increased filling resistance, excessive tachycardia (above 170/minute), myocardial failure, or relative nonresponsiveness to amyl nitrite.

The Value of Amyl Nitrite in the Diagnosis of Pulmonary Stenosis. The softening of the systolic murmur, the drop in right ventricular systolic pressure, and the increased right-to-left shunt following amyl nitrite inhalation may be thought to invalidate previous claims that this drug is of value in differentiating pulmonary stenosis from Fallot's tetralogy (Vogelpoel et al., 1959). Amyl nitrite is still of great value in differentiating mild and moderately severe pulmonary stenosis from cyanotic or acyanotic Fallot's tetralogy. When this diagnostic problem arises, the right ventricular systolic pressure in pulmonary stenosis is usually at or below systemic level so that it is not usually associated with excessive right ventricular hypertrophy. Consequently the increase in right ventricular systolic pressure and intensification of the systolic murmur contrasts sharply with the drop in right ventricular pressure and the softening and shortening of the murmur that occurs in Fallot's tetralogy. The patient with severe pulmonary stenosis and a "muscle-bound" right ventricle is usually easily distinguished from Fallot's tetralogy without resort to amyl nitrite. Thus, the length and shape of the systolic murmur will usually be different (Vogelpoel and Schrire, 1960) and the right ventricular systolic pressure will exceed systemic. Although the right ventricular pressure falls after amyl nitrite, the systolic pressures are widely separated and behave independently (Fig. 6).

\section{SUMMARY}

A study has been made to determine criteria by which the excessively hypertrophied, "musclebound" right ventricle in pulmonary stenosis with intact ventricular septum can be diagnosed before operation. Nineteen patients who underwent complete open pulmonary valvotomy were selected for study: 11 had no significant infundibular obstruction after surgery, and 8 had gross hypertrophy resulting in severe secondary infundibular obstruction, immediately after valvotomy. Ventricles associated with this complication were termed "muscle-bound".

The following new observations were made and should prove helpful in the pre-operative diagnosis. When the right ventricle was "muscle-bound", the jugular or right atrial tracing showed a very slow " $y$ " descent and shallow or even absent " $y$ " trough after the delayed " $v$ " wave, in the absence of tachycardia. Of greater significance was the paradoxical softening of the systolic murmur and drop in right ventricular systolic pressure during the tachycardia phase after amyl nitrite inhalation.

By contrast, when the right ventricle was not "muscle-bound", a more normal right atrial pressure pulse was found and the right ventricle was capable of promptly increasing its systolic pressure and ejection velocity during the tachycardia phase after amyl nitrite.

In severe pulmonary stenosis two major factors are believed to limit the diastolic reserve capacity of the right ventricle. Diastolic filling time is shortened by the gross prolongation of systole and diastolic filling resistance is increased by the hypertrophied muscle. The latter is reflected by the " $y$ " descent and " $y$ " trough of the right atrial pulse, while amyl nitrite tests the ability of the right ventricle to increase its output during a severely curtailed filling time. The greater and/or stiffer the hypertrophied muscle mass, the greater the impedance to filling, and the greater the handicap during tachycardia. The non-"muscle-bound" right ventricle is able to increase its output, whereas the output drops at relatively slow rates when the right ventricle is severely "muscle-bound". The "muscle-bound" right ventricle is conceived as having great ejectile power, but being bulky, stiff, and slow to dilate, has greatly increased diastolic filling resistance. It functions well when adequate diastolic filling is ensured by slow heart-rate, but its performance drops off severely during tachycardia. 
We wish to thank members of the staff of Groote Schuur Hospital for referring patients for investigation and the Superintendent, Dr. J. G. Burger, for permission to publish. We are indebted to Professor C. N. Barnard and his surgical team for without their skill and co-operation this paper could not have been written.

We gratefully acknowledge the assistance of Dr. A. Swanepoel and other colleagues in the Cardiac Clinic, and that of our technicians, Mr. L. W. Piller, Miss S. Joseph, and Miss M. Cross. We also wish to thank the nursing staff and Mrs. C. M. Hall for her secretarial work.

Part of the expenses of this work has been defrayed by grants received from the Council for Scientific and Industrial Research and the City Council of Cape Town.

\section{REFERENCES}

Beck, W., Schrire, V., Vogelpoel, L., Nellen, M., and Swanepoel, A. (1961). Hemodynamic effects of amyl nitrite and phenylephrine on the normal human circulation and their relation to changes in cardiac murmur. Amer. J. Cardiol., 8, 341.

Blount, S. G., Jr., van Elk, J., Balchum, O. J., and Swan, H. (1957). Valvular pulmonary stenosis with intact ventricular septum. Clinical and physiologic response to open valvuloplasty. Circulation, $15,814$.

Brock, R. (1955). Control mechanisms in the outflow tract of the right ventricle in health and disease. Guys' Hosp. Rep., 104, 356.

- (1957). The Anatomy of Congenital Pulmonary Stenosis. Cassell, London.

(1961). The surgical treatment of pulmonary stenosis. Brit. Heart J., 23, 337.

Honig, C. R., Tenney, S. M., and Gabel, P. V. (1960). The mechanism of cardiovascular action of nitroglycerine. An example of integrated response during the unsteady state. Amer. J. Med., 29, 910.

Johnson, A. M. (1959). Hypertrophic infundibular stenosis complicating simple pulmonary valve stenosis. Brit. Heart J., 21, 429.

_ (1962). Impaired exercise response and other residua of pulmonary stenosis after valvotomy. Brit. Heart J., 24, 375 .

Kirklin, J. W., Connolly, D. C., Ellis, F. H., Burchell, H. B., Edwards, J. E., and Wood, E. H. (1953). Problems in the diagnosis and surgical treatment of pulmonic stenosis with intact ventricular septum. Circulation, 8, 849.

Rushmer, R. F. (1961). Cardiovascular Dynamics. Saunders, Philadelphia.

Vogelpoel, L., and Schrire, V. (1960). Auscultatory and phonocardiographic assessment of pulmonary stenosis with intact ventricular septum. Circulation, 22, 55.

- - - Beck, W., Nellen, M., and Swanepoel, A. (1962). Variations in the response of the systolic murmur to vasoactive drugs in ventricular septal defect, with special reference to the paradoxical response in large defects with pulmonary hypertension. Amer. Heart J., 64, 169.

,$- \frac{1}{-}$, Nellen, M., and Swanepoel, A. (1959). The use of amyl nitrite in the differentiation of Fallot's tetralogy and pulmonary stenosis with intact ventricular septum. Amer. Heart J., 57, 803.

Watson, H., Pickard, C., Lowe, K. G., and Hill, I. G. W. (1960). Cine-angiocardiographic studies of the outflow tract in isolated pulmonary valvular stenosis. Brit. Heart J., 22, 706.

Wiggers, C. J. (1949). Physiology in Health and Disease, 5th ed. Kimpton, London. 